\title{
DISEÑO Y EXPERIMENTACIÓN DE UN CURSO MOOC PARA CUIDADORES INMIGRANTES DE MAYORES: PROYECTO IENE7
}

\author{
Ana Morales Montoya \\ moralesmontoyaana@gmail.com \\ Remedios López Liria \\ rll040@ual.es \\ David Padilla Góngora \\ dpadilla@ual.es \\ Patricia Rocamora Pérez \\ rocamora@ual.es \\ Pilar Díaz López \\ pilardiaz@crecimientohumano.com \\ José Manuel Aguilar Parra \\ jmaguilar@ual.es \\ Universidad de Almería
}

\section{RESUMEN}

Introducción: Desde septiembre de 2017, el grupo de investigación Humanidades-498 (Intervención psicológica en desarrollo, educación y orientación) de la Universidad de Almería, está trabajando en un proyecto europeo junto a otros cuatro socios (Rumanía, Reino Unido y dos comunidades de Italia) en la creación de un modelo adecuado de educación dirigido a cuidadores informales de personas mayores que reciben atención domiciliaria. Este modelo será el fundamento teórico que apoyará la creación de un currículo adecuado, con objetivos, contenidos y materiales adaptados a estos grupos de cuidadores que en muchas ocasiones son grupos desfavorecidos (inmigrantes, personas sin formación o en riesgo de exclusión social, etc.). Las herramientas y recursos de aprendizaje elaborados se introducirán en una plataforma desarrollada para contener un Curso MOOC (curso online gratuito y disponible en la red para toda la comunidad internacional traducido en cuatro idiomas) y una aplicación Smartphone de licencia gratuita. El objetivo principal de este proyecto es ofrecer formación para los cuidadores informales, mejorar sus oportunidades de trabajo, fortalecer su posición en el mercado laboral y aumentar la calidad en la atención a los mayores en diversos países europeos. Metodología: se desea desarrollar y pilotar un Curso Online (generalmente llamados MOOC) construido con un enfoque pedagógico innovador de coaprendizaje y basa- 


\section{DISEÑO Y EXPERIMENTACIÓN DE UN CURSO MOOC PARA CUIDADORES INMIGRANTES DE MAYORES: PROYECTO IENE7}

do en las tecnologías digitales como facilitadores del proceso de enseñanza. Dirigido a personas con diferentes cualificaciones profesionales: auxiliares de enfermería, cuidadores formales e informales, familias, especialista en cuidados geriátricos...Se trata de crear una comunidad online en la plataforma de aprendizaje para compartir intereses comunes y promover valores e interacciones positivas en el cuidado de las personas mayores. Resultados: nuestra propuesta aborda principalmente a los grupos desfavorecidos de inmigrantes, muchos de ellos no cualificados, para mejorar su comunicación profesional, intercultural y social con el fin de aumentar su profesionalidad, empleabilidad, fomentar su participación activa y cambiar las actitudes estigmatizadas de la sociedad y algunas instituciones. Promueve métodos y pedagogías innovadoras centradas en el desarrollo de una educación abierta e innovadora a través del uso de las TICS, que podría ser ampliamente utilizadas en otros contextos. Conclusiones: este proyecto desea mejorar el acceso, la participación y el aprendizaje de grupos desfavorecidos, prioridad horizontal de los programas europeos ERASMUS +.

Palabras clave: cuidadores informales; inmigrantes; M00C; aplicación; educación online

\section{ABSTRACT}

Design and experimentation of a mooc course for immigrant carers for older persons: iene7 project.

Introduction: Since September 2017, the research group Humanities-498 (Psychological intervention in development, education and guidance) of the University of Almeria, is working on a European project together with four other partners (Romania, United Kingdom and two communities of Italy) in the creation of an appropriate model of education for informal caregivers of the elderly who receive home care. This model will be the theoretical foundation that will support the creation of an adequate curriculum, with objectives, content and materials adapted to these groups of caregivers who are often disadvantaged groups (immigrants, people without education or at risk of social exclusion, etc.) . The tools and learning resources developed will be introduced in a platform developed to contain a MOOC Course (free online course available on the net for the entire international community translated into four languages) and a free license Smartphone application. The main objective of this project is to provide training for informal caregivers, improve their job opportunities, strengthen their position in the labor market and increase the quality of care for the elderly in various European countries. Methodology: you want to develop and pilot an Online Course (generally called MOOC) built with an innovative pedagogical approach to co-learning and based on digital technologies as facilitators of the teaching process. Aimed at people with different professional qualifications: nursing assistants, formal and informal caregivers, families, geriatric care specialist ... It is about creating an online community in the learning platform to share common interests and promote values and positive interactions in the care of old people. Results: our proposal mainly addresses the disadvantaged groups of immigrants, many of them unskilled, to improve their professional, intercultural and social communication in order to increase their professionalism, employability, encourage their active participation and change the stigmatized attitudes of society and some institutions. It promotes innovative methods and pedagogies focused on the development of an open and innovative education through the use of ICT, which could be widely used in other contexts. Conclusions: this project aims to improve access, participation and learning of disadvantaged groups, horizontal priority of the ERASMUS + European programs.

Keywords: informal caregivers; immigrants; MOOC; application; online education

\section{INTRODUCCIÓN:}

En las últimas décadas, los cambios demográficos en las sociedades desarrolladas están conduciendo a un aumento significativo del envejecimiento de la población. En España este envejeci- 
miento puede ser considerado como un fenómeno dinámico, dada su singular velocidad en comparación con otros países también evolucionados. Las dos fuerzas que lo explican son la caída de la fecundidad y el avance de la longevidad, provocando esta última un aumento de las enfermedades crónico degenerativas y, consecuentemente de la dependencia. Se puede entender por "dependencia” el hecho de no poder valerse por sí mismo en algún grado (Serrano-Ortega, Frías-Osunaa, Recio-Gómez, \& Del-Pino-Casado, 2015).

En estos países, los cuidados de larga duración prestados a las personas mayores provenían hasta el momento del entorno familiar. Siendo la mujer fundamentalmente la que se encargaba, asumiendo el papel de cuidadora principal (Garcés, Carretero, Ródenas, \& Vivancos, 2010)

La caída de la natalidad, el aumento de la esperanza de vida, la incorporación de la mujer al mercado laboral y la tendencia hacia la privatización de la política social, son algunos de los factores más llamativos que han condicionado la demanda de cuidados de las personas mayores y de aqueIlos que ofrecen dicha asistencia. Al mismo tiempo, es apreciable la influencia en la última década, de la creciente internacionalización del mercado de trabajo, acompañada de una aceleración de los flujos migratorios que eligen España, como destino para su proyecto de vida, con dos rasgos básicos, su feminización y su carácter irregular. Todas estas condiciones han favorecido el reemplazo del cuidador familiar no remunerado de personas mayores, por cuidadoras inmigrantes a las se ofrece empleo para realizar estas tareas de atención personal (Rodríguez Rodríguez et al., 2010).

El $90 \%$ de los cuidadores de personas mayores remunerados en España son inmigrantes, se estima que puede haber 200.000 personas inmigrantes que desempeñan esta labor (Corregido, 2012). Según datos de la afiliación a la Seguridad Social ofrecidos por el Ministerio de Trabajo en junio de 2006, el 67,3\% de las personas incluidas en el régimen especial de servicio doméstico eran extranjeras (Martínez Buján, 2011)

La existencia de personas dependientes supone la necesidad de otras personas que las ayuden, esto se ha reconocido en la "Ley de Promoción de la autonomía personal y atención a las personas en situación de dependencia" (Ley 39/2006), que define los "cuidados de larga duración" como el "cuarto pilar del estado de bienestar" (Rogero, 2009). El cuidado que reciben las personas dependientes puede proceder de sus familiares, de personal sanitario o de ambos. Se define el "apoyo informal" como el "cuidado y atención fundamentalmente ofrecido por familiares y allegados, o por otros agentes que lo dispensan de manera altruista a las personas que presentan algún grado de discapacidad o dependencia" (Rogero García, 2010).

Por otro lado, entendemos por "cuidado formal" a las tareas que oferta un profesional especializado y que van más allá de las propias capacidades de las personas para cuidar de sí mismas o de los demás. Existen dos tipos de cuidados formales: el que tiene lugar en las instituciones y el que se contrata a través de las familias (Rodríguez-Rodríguez, 2005).

Todo proceso de cuidado requiere de una formación para aumentar la eficacia de los cuidadores y corregir las deficiencias que se puedan generar en este trabajo por desconocer un adecuado desempeño. Al hablar de "formación dirigida al cuidador", en ocasiones se hace referencia a la orientación, supervisión y valoración que los profesionales sanitarios, fundamentalmente de enfermería, desempeñan con la educación sanitaria o "la educación para la salud" que ofrecen en Centros de Salud, asociaciones, o los domicilios (Martínez Qués, 2007). Diversas investigaciones realizadas en nuestro país, describen en el perfil del cuidador informal un nivel de estudios que suele corresponder a un nivel bajo (Zambrano-Domínguez \& Guerra-Martín, 2012).

Generalmente, los cuidadores informales no poseen la suficiente formación en cuanto a cuidados básicos generales (Prieto Rodríguez, Gil García, Heierle Valero, \& Frías Osuna, 2002), ni de los procesos patológicos de las personas mayores a las que cuidan (Torres Egea, Ballesteros Pérez, \& 


\section{DISEÑO Y EXPERIMENTACIÓN DE UN CURSO MOOC PARA CUIDADORES INMIGRANTES DE MAYORES: PROYECTO IENE7}

Sánchez Castillo, 2008), siendo esta formación muy importante para asegurar una provisión de cuidados de calidad, en tanto que afecta a la salud de la persona cuidada y repercute sobre la suya propia.

Debido a las cuestiones aquí descritas, el grupo de investigación Humanidades-498 de la Universidad de Almería, está desarrollando un proyecto europeo de intervención titulado "Mejorar la comunicación, las competencias interculturales y sociales para los extranjeros e inmigrantes, que trabajan como cuidadores de personas mayores en Europa: proyecto IENE 7". En dicho proyecto participan cinco socios de cuatro países (Rumanía, Italia, Reino Unido y España), habiendo sido concedido por la Comisión Europea en el marco de las acciones K2 "cooperación para la innovación y el intercambio de buenas prácticas".

El Proyecto IENE 7 tiene como objetivo básico cubrir la ausencia de formación para cuidadores de personas mayores. Formar a los empleados inmigrantes de dentro y fuera de la Unión Europea en este cuidado domiciliario, con una adecuada comunicación, competencia intercultural y habilidades sociales con el fin de mejorar sus oportunidades de empleo, fortalecer su posición en el mercado laboral y el aumento de la calidad en la atención ofrecida a las personas mayores de los países participantes y el resto de la Unión Europea.

\section{METODOLOGIA Y RESULTADOS ESPERADOS}

Las actividades del proyecto se han iniciado con la revisión de la literatura científica sobre las necesidades de esta muestra de población de cuidadores y la creación de un modelo de formación nuevo e innovador basado en el modelo PPT/ IENE (Papadopoulos, Tilki, Taylor, 1998; Papadopoulos, 2006).

El proyecto está utilizando un modelo europeo para la educación intercultural de enfermeras y profesionales de la salud (modelo PTT/ IENE), fundamento teórico que apoyará la creación de un currículo adecuado para una formación adaptada a los grupos de inmigrantes que trabajan en el cuidado de ancianos en el hogar; módulos y temas basados en las competencias establecidas en las cuatro áreas que constituyen este modelo de entrenamiento (Papadopoulos, Tilki, Taylor, 1998; Papadopoulos, 2006).

Las actividades del proyecto se están agrupando en varios paquetes de trabajo específicos como son la gestión, resultados intelectuales, eventos, evaluación, difusión y valorización. Cada producto tiene un líder, que coordina el trabajo para lograr las actividades planificadas.

El "Currículo" es la actividad que está asignada al grupo de investigación Humanidades-498 y que guiará los objetivos de aprendizaje, los resultados y las actividades del curso. Para cada tema, se creará una herramienta con información dividida en varias secuencias, organizadas de forma atractiva, con enlaces a recursos electrónicos, vídeos de youtube y animaciones. Las herramientas y recursos de aprendizaje elaborados se introducirán en una plataforma desarrollada para contener un Curso MOOC (curso online gratuito y disponible en la red para toda la comunidad internacional traducido en cuatro idiomas) y una aplicación Smartphone de licencia gratuita.

Antes de poner a prueba la aplicación MO0C, se organizarán conjuntamente talleres prácticos con los socios de los cuatro países para capacitar a instructores estatutarios y voluntarios sobre cómo usarla y cómo llevar a cabo el proceso de enseñanza-aprendizaje con los participantes.

Durante la fase de validación del MOOC, necesitamos contar con el apoyo de colaboradores (de comunidades locales, organizaciones educativas, organizaciones de mayores, agencias de colocación laboral, proveedores de formación), y se realizará el pilotaje del curso con un mínimo de 100 cuidadores de mayores. Se espera que, al menos la mitad de los participantes de este curso MOOC, sean de grupos desfavorecidos (con diferencias culturales, refugiados, problemas de salud, proble- 
mas geográficos y sociales). Finalmente, tras completar todos los módulos, los participantes realizarán los cuestionarios de evaluación de las unidades temáticas, siendo calificados según las competencias logradas y se emitirán certificados para aquellas personas que hayan finalizado el curso de forma satisfactoria.

También se va a diseñar un cuestionario de evaluación del curso, donde el participante pueda dar una valoración de la metodología y los contenidos del curso, así como del currículo y los recursos de aprendizaje utilizados.

Después de la fase de validación, se desea que esta metodología y el contenido del curso estén disponibles durante mucho tiempo en la plataforma del proyecto y se puedan utilizar junto con el modelo de formación y el currículo diseñado, por aquellas personas que lo deseen de forma gratuita, ofreciendo la posibilidad de ser un ejemplo para el desarrollo de nuevos cursos online dirigidos a otros sectores sociales y diferentes contextos.

Entre los resultados esperados:

- Se desea que el modelo de capacitación y el currículo estén disponibles en la plataforma web del proyecto, de modo que puedan ser descargados y utilizarse ofreciendo la oportunidad de capacitar o entrenar a distintos profesionales; ser un ejemplo de buena práctica para el proceso de enseñanza-aprendizaje.

- Se dispondrá de diferentes herramientas para el aprendizaje de los contenidos disponibles en la plataforma del curso en cuatro idiomas.

- Los recursos de capacitación, aprendizaje y evaluación estarán en la plataforma del curso MOOC, para que puedan ser utilizados tanto por estudiantes, como por cuidadores de mayores 0 profesionales. Dichos recursos podrán ser configurados y actualizados, ya que la plataforma está gestionada por el sistema Comntent.

- Desde la página web del proyecto también se podrá descargar una aplicación Smartphone, y los materiales de aprendizaje podrán ser accesibles a través de tablets o móviles.

\section{DISCUSIÓN Y CONCLUSIONES}

El envejecimiento de la población es uno de los rasgos demográficos que caracterizan al desarrollo de las sociedades contemporáneas y, además, uno de los principales desafíos del siglo XXI. El aumento de la edad de la población está asociado generalmente, a una pérdida de capacidades funcionales, de la que se deriva la necesidad y la provisión de cuidados por una tercera persona como apoyo para la realización de las actividades básicas de la vida diaria.

El problema social de la dependencia no es nuevo en España. Lo novedoso es la dimensión que ha alcanzado el problema, debido a factores sociodemográficos como el envejecimiento de la población, los cambios en el funcionamiento familiar y el aumento de la longevidad, entre otros. Al igual que ha sucedido en otros procesos sociales (por ejemplo, los problemas económicos o los problemas de fertilidad), lo característico de todo esto es la intensidad que han recibido estos fenómenos y la rápida incorporación al debate científico y político (Pérez Ortiz, 2003).

La existencia de un modelo de cuidados basado en la aportación de la familia, ha permitido hasta ahora cuidar en el domicilio a la persona dependiente con ciertos niveles de discapacidad, pero los cambios sociales hacen que esa situación se esté desmoronando. En la actualidad se observa como las necesidades que tradicionalmente cubría la familia están quedando al descubierto. Los familiares, para no desentenderse de los cuidados, y ante la falta de recursos sociosanitarios de proximidad buscan otro tipo de ayuda generalmente en el mercado de trabajo, recurriendo a la contratación de mujeres inmigrantes para que asuman estos cuidados familiares (Galiana- Gómez de Cádiz, De la Cuesta- Benjumea, \& Donet- Montagut, 2008). De este modo, el cuidado brindado por inmigran- 


\section{DISEÑO Y EXPERIMENTACIÓN DE UN CURSO MOOC PARA CUIDADORES INMIGRANTES DE MAYORES: PROYECTO IENE7}

tes se presenta como un importante recurso a la dependencia, siendo éstos los nuevos actores dentro del entramado del sistema de atención a la salud en España (Corregido, 2012). Lo que sucede, es que al confluir la inmigración y el trabajo doméstico por mujeres frente al cuidado habitual familiar, esta actividad se encuentra teñida de una connotación de vulnerabilidad extrema.

La atención que deben prestar es compleja, en cuanto a que las cuidadoras inmigrantes deben saber manejar medicación, cuidados básicos, higiene, alimentación, en una cultura y sistema sanitario que les son ajenos. Al mismo tiempo deben ser nexo de comunicación con los equipos de salud con los que no suelen estar familiarizadas. Trabajan más duro cuanto mayor es la situación de dependencia, apareciendo con frecuencia sentimientos de distrés o carga, que están influenciados por el tipo de enfermedad o nivel de discapacidad de la persona mayor (Gallart Fernández-Puebla, 2007).

Las nuevas formas de cuidado suponen un reto para la sociedad ya que abren numerosas líneas de estudio e investigación. A la hora de planificar servicios de salud y de promover políticas que apoyen el cuidado familiar, es necesario reconocer la implicación de otros actores de fuera de la familia. Si se obvia que en el cuidado familiar cada vez más intervienen las cuidadoras inmigrantes, éstas estarían quedando excluidas hasta el momento de los estudios y acciones realizadas. Reconocer la importancia de estas nuevas cuidadoras en el apoyo a las familias con personas dependientes supone a los investigadores un gran campo de actuación (Bover et al., 2015).

Como conclusiones, este proyecto se está centrando en crear un nuevo e innovador modelo de formación, que apoyará la creación de un currículo adecuado, con objetivos, contenidos y materiales adaptados a estos grupos de cuidadores que en muchas ocasiones son grupos desfavorecidos (inmigrantes, personas sin formación o en riesgo de exclusión social, etc.). Las herramientas y recursos de aprendizaje elaborados se introducirán en una plataforma desarrollada para contener un Curso MOOC (curso online gratuito y disponible en la red para toda la comunidad internacional traducido en cuatro idiomas), construido con un enfoque pedagógico innovador de coaprendizaje y basado en las tecnologías digitales como facilitadores del proceso de enseñanza.

Las actividades de este proyecto se han planificado para que tengan una duración de dos años, esperándose que el curso de formación online generado ofrezca una mejor preparación para los cuidadores formales e informales domiciliarios, cuestión que sin duda redundaría en impacto tanto social, como sobre las instituciones sociosanitarias encargadas de proveer servicios de calidad. Este proyecto desea mejorar el acceso, la participación y el aprendizaje de grupos desfavorecidos, prioridad horizontal de los programas europeos ERASMUS +.

\section{REFERENCIAS}

Bover, A., Taltavull, J. M., Gastaldo, D., Luengo, R., Izquierdo, M. D., Juando-Prats, C., ... Robledo, J. (2015). Calidad de vida de trabajadoras inmigrantes latinoamericanas como cuidadoras en España. Gaceta Sanitaria, 29(2), 123-126. https://doi.org/10.1016/j.gaceta.2014.09.009

Corregido, D. J. (2012). Género y trayectorias migratorias en época de crisis. Papers, 97(3), 523-540. https://doi.org/10.5565/rev/papers/v97n3.438

Galiana- Gómez de Cádiz, M. J., De la Cuesta- Benjumea, C., \& Donet- Montagut, T. (2008). Cuidadoras inmigrantes: características del cuidado que prestan a la dependencia. Enfermería Clínica, 18(5), 269-272. https://doi.org/10.1016/S1130-8621(08)72386-6

Gallart Fernández-Puebla, A. (2007). Sobrecarga del cuidador inmigrante no profesional formal. Universitat Internacional de Catalunya.

Garcés, J., Carretero, S., Ródenas, F., \& Vivancos, M. (2010). The care of the informal caregiver's burden by the Spanish public system of social welfare: A review. Archives of Gerontology and 
Geriatrics, 50(3), 250-253. https://doi.org/10.1016/j.archger.2009.04.011

Martínez Buján, R. (2011). La reorganización de los cuidados familiares en un contexto de migración internacional. Cuadernos de Relaciones Laborales, 29(1), 93-123. https://doi.org/10.5209/rev_CRLA.2011.v29.n1.4

Martínez Qués, Á. A. (2007). La Dependencia, los Cuidadores y la Necesidad de Formación. Enfermería Docente, 12(3), 241-251.

Papadopoulos I, Tilki M and Taylor G (1998): Transcultural Care: A guide for Health Care Professionals. Quay Books. Wilts. (ISBN 1-85642-051 5)

Papadopoulos I (Ed): (2006): Transcultural Health and Social Care: Development of Culturally Competent Practitioners. Churchill Livingstone Elsevier. Edinburgh.

Pérez Ortiz, L. (2003). Construcción social de la vejez: el sexo y la dependencia. Revista Española de Geriatría Y Gerontología, 38(6), 308-310.

Prieto Rodríguez, M. Á., Gil García, E., Heierle Valero, C., \& Frías Osuna, A. (2002). La perspectiva de las cuidadoras informales sobre la atención domiciliaria. Un estudio cualitativo con ayuda de un programa informático. Revista Espanola de Salud Publica, 76(5), 613-625. https://doi.org/10.1590/S1135-57272002000500021

Rodríguez Rodríguez, V., Martín Coppola, E., Marcu, S., Ramos Espina, S., Rojo Pérez, F., FernándezMayorala Fernández, G., ... Rogero García, J. (2010). Inmigración y cuidados de mayores en los hogares de la Comunidad de Madrid.

Serrano-Ortega, N., Frías-Osunaa, A., Recio-Gómez, J. M., \& Del-Pino-Casado, R. (2015). Diseo y validación de una escala para la medición de la dedicación al cuidado en personas cuidadoras de mayores dependientes (DeCuida). Atencion Primaria, 47(9), 589-595.

Torres Egea, M. P., Ballesteros Pérez, E., \& Sánchez Castillo, P. D. (2008). Programas e intervenciones de apoyo a los cuidadores informales en España. Gerokomos, 19(1), 9-15. https://doi.org/10.4321/S1134-928X2008000100002

Zambrano-Domínguez, E. M., \& Guerra-Martín, M. D. (2012). Formación del cuidador informal: relación con el tiempo de cuidado a personas dependientes mayores de 65 años. Aquichan, 12(3), 241-251. https://doi.org/10.5294/aqui.2012.12.3.3 
